\title{
The American Phytopathological Society Elected and Appointed Officers, Representatives, and Committees for 2003
}

Council

President: J. Fletcher

President-Elect: G. C. Bergstrom

Immediate Past President: S. A. Slack

Vice President: J. D. MacDonald

Secretary: C. A. Ishimaru

Treasurer: J. L. Sherwood

Treasurer-Elect: E. L. Stromberg

Editor-in-Chief, Phytopathology: C. C. Mundt

Editor-in-Chief, Plant Disease: A. R. Biggs

Editor-in-Chief, Molecular Plant-Microbe Interactions: H. P. Spaink

Editor-in-Chief, Phytopathology News: S. A. Johnston

Editor-in-Chief, APS Press: R. C. Ploetz

Senior Councilor-at-Large: J. H. Andrews

Intermediate Councilor-at-Large: R. R. Belanger

Junior Councilor-at-Large: M. A. Ellis

Councilor of the Caribbean Division: J. K. Brown

Councilor of the North Central Division: R. D. Martyn

Councilor of the Northeastern Division: B. J. Christ

Councilor of the Pacific Division: M. E. Matheron

Councilor of the Potomac Division: A. Grybauskas

Councilor of the Southern Division: A. K. Culbreath

Ex officio: APS Foundation Rep.: D. E. Mathre, Executive VP.: S. C. Nelson

\section{Representatives}

American Association for the Advancement of Science: D. Gilchrist

American Institute of Biological Sciences: J. D. MacDonald

American Type Culture Collection: G. H. Lacy

Coalition for Funding Agricultural Research: S. A. Tolin, J. L. Sherwood

Council for Agricultural Science and Technology: B. J. Jacobsen

International Society of Arboriculture: R. J. Stipes

International Society for Plant Pathology: Chair: A. M. Alvarez. G. S. Abawi, R.

A. Bennett, K. F. Cardwell, L. V. Madden, J. Moyer, C. C. Mundt, B. W.

Pennypacker, L. Sequeira, P. S. Teng, A. H. van Bruggen, R. S. Zeigler

International Union of Microbiological Societies: C. J. Baker

Mycological Society of America: C. M. Stiles

National Council for Science and the Environment: T. A. Kucharek

National Research Council, Commission on Life Sciences: C. A. Ishimaru

National Research Council, CLS/Board of Agriculture: G. C. Bergstrom

Society of Environmental Toxicology and Chemistry: M. Simini

Society of Nematologists: E. L. Davis

APS Historian: P. D. Peterson, Jr.

APS Foundation, Board of Directors

Chair: D. E. Mathre. Vice Chair: P. R. Sellers. Secretary-Treasurer: J. R. French. D. A. Cooksey, C. J. D’Arcy, W. E. Fry, G. L. Schumann, Ex officio: J. L. Sherwood, Z. Kanaan-Atallah, S. C. Nelson

Office of Electronic Communication (OEC) Board

Director: J. B. Ristaino. J. S. Batten, D. T. Beadle, C. T. Bull, A. O. Charkowski, W. Chun, D. M. Eastburn, N. Grunwald, G. Hughes, E. C. Tedford

\section{Office of International Programs (OIP) Advisory Board}

Director: G. S. Abawi. L. L. Black, W. M. Brown, K. F. Cardwell, G. A. Forbes, E. R. French, J. H. McBeath, R. C. Ploetz, N. W. Schaad, J. R. Steadman, R. S. Zeigler, Ex officio: W. E. Fry, S. C. Nelson

Education: Chair: K. F. Cardwell. J. E. Bowman, N. Grunwald, B. Guo, D. M. Huber, J. McBeath, T. Mew, S. A. Rizvi, S. L. von Broembsen

Policies and Programs: Chair: R. Zeigler, G. S. Abawi, L. L. Black, K. F. Cardwell, H. J. Dubin, B. K. Duffy, N. W. Schaad, J. R. Steadman, F. H. Tainter

Research: Chair: N. W. Schaad. N. A. Altier, J. K. Brown, C. A. Clark, G. A. Forbes, E. R. French, V. S. Malik, D. C. Sands, J. P. Wilson

Service: Chair: J. R. Steadman. M. Babadoost, W. M. Brown, C. Estevez deJensen, M. A. Pastor-Corrales, L. Marques, S. A. Miller, R. C. Ploetz, R. Soufi

Office of Industry Relations (OIR) Board

Director: C. M. Becker. Recording Director: M. R. Miles, L. Fought, R. P. Kaiser, M. D. Lees, J. L. Riggs, Z. Shi, M. G. Tiffany, Industry Liaison: C. L. Palmer, Private Practice Liaison: W. T. Cobb. Sus. Assoc. Liaison: K. Arthur
Office of Public Affairs and Education (OPAE) Advisory Board

Director: G. L. Tylka. Director-Elect: D. J. Jardine, Communications Coordinator: M. L. Daughtrey, K. Cardwell, C. R. Curtis, M. A. Ellis, J. E. Partridge, A. Tally, PPB Chair: J. L. Sherwood, APSnet Feature Editor: D. Eastburn, APS President: J. Fletcher, Ex-officio: C. Ash, M. Bjerkness

Public Policy Board (PPB)

Chair: J. L. Sherwood. J. Amador, O. W. Barnett, G. C. Bergstrom, N. B. Carroll, J. Fletcher, S. E. Gold, J. E. Leach, J. D. MacDonald, D. C. McGee, R. E. Stuckey, Ex-officio: M. Bjerkness, S. C. Nelson, G. L. Tylka

Scientific Programs Board

Director: E. N. Rosskopf. Section Chairs: S. T. Adkins, R. A. Bennett, C. T. Bull, S. M. Douglas, M. L. Elliott, J. E. Lundquist. J. H. Andrews, G. C. Bergstrom, R. M. Davis, J. D. MacDonald, G. L. Schumann, Ex-officio: Staff

APS Publications Board

Chair: R. C. Ploetz. A. R. Biggs, B. I. Hillman, S. A. Johnston, T. D. Murray, G. L. Schumann, H. P. Spaink

\section{Editors of APS Press}

Editor-in-Chief: R. C. Ploetz. EIC-Elect: R. C. Gergerich. Senior Editors: C. M. Becker, M. B. Dickman, D. Gonsalves, G. Hughes, J. B. Jones, G. W. Moorman, T. J. Volk, Section Editors: A. M. Alvarez, M. Putnam. Ex officio: J. L. Sherwood, Staff

APS Press Editorial Boards and Committees

Biological and Cultural Tests: Senior Editor: S. C. Bost. Section Editors: R. L. Bowden, G. J. Holmes, T. L. Kirkpatrick, J. F. Powell

Fungicide and Nematicide Tests: Senior Editor: R. E. Baird. Section Editors: W. O. Cline, D. S. Egel, A. Henn, S. N. Jeffers, R. C. Kemerait, W. Kirk, R. J. McGovern, P. S. McManus, J. S. Miller, J. R. Rich, L. E. Trevathan, W. Uddin

Illustrations of Plant Pathogens and Diseases: Chair: T. J. Volk, C. S. Rothrock

Phytopathological Classics: C. S. Griffith

Standardization of Common Names of Plant Diseases: Chair: P. F. Bertrand. Vice Chair: TBD. Immediate Past Chair: M. Putnam. A. Grybauskas, E. V. Podleckis

Editors of MOLECULAR PLANT-MICROBE INTERACTIONS

Editor-in-Chief: H. P. Spaink. Senior Editors: C. A. Boucher, J. Glazebrook, F. Govers, D. Haas, M. J. Harrison, J. W. Kronstad, J. E. Schoelz, J. Stougaard, M. Udvardi, V. M. Williamson. Associate Editors: D. L. Beck, A. Becker, N. J. Brewin, J. P. Carr, Z. Chen, L. Ciuffetti, J. V. Cullimore, J. Dangl, M. Djordjevic, X. Dong, D. Expert, O. Geiger, G. Gheysen, S. E. Gold, M. Grant, S. Kamoun, B. N. Kunkel, K. Lambert, S. A. Lommel, T. McDermott, J. McDowell, Y. Moenne-Loccoz, P. Müller, D. Noel, T. Nuernberger, M. Parniske, K. Pawlowski, S. Perotto, J. M. Salmeron, F. Sanchez, H. B. Scholthof, S. C. Somerville, J. Sweigard, K. Szczyglowski, N. J. Talbot, I. K. Toth, J. Van Kan, N. D. Young

\section{Editors of PHYTOPATHOLOGY}

Editor-in-Chief: C. C. Mundt. Senior Editors: J. C. Correll, M. J. Davis, S. E. Gold, L. L. Kinkel, R. P. Larkin, F. N. Martin, P. V. Oudemans, B. H. Ownley, H. Scherm, S. M. Schneider, H. B. Scholthof, D. C. Stenger, J. P. Wilson. Associate Editors: J. Borneman, M. K. Clayton, J. N. Culver, C. M. Deom, S. W. Ding, C. M. Fauquet, R. D. Frederick, K. A. Garrett, J. S. Gerik, R. W. Jones, R. R. Martin, P. S. McManus, J. F. Murphy, E. B. Nelson, D. Panaccione, T. Powers, D. Rigling, D. M. Rizzo, A. Rowhani, K.-B. Scholthof, C. D. Smart, V. O. Stockwell, L. Tredway, F. van den Bosch,W. M. Wintermantel, C. L. Xiao, X. Xu

\section{Editors of PLANT DISEASE}

Editor-in-Chief: A. R. Biggs. Senior Editors: K. L. Bowen, P. D. Colyer, L. L. Domier, M. L. Gleason, T. C. Harrington, M. K. Hausbeck, P. J. Landschoot, C.-H. Liao, G. P. Munkvold, J. C. Rupe, D. Seifers, K. L. Stevenson. Feature Editor: N. A. Tisserat. Disease Notes Assigning Editor: A. P. Keinath. Focus Editor: T. Kommedahl. Associate Editors: J. O. Becker, P. Bonello, E. D. De Wolf, K. Eastwell, F. A. Gray, H. M. Griffiths, J. R. Hartman, R. M. Hunger, D. A. Inglis, L. L. Kinkel, S. T. Koike, G. Kuldau, D. S. Marshall, B. D. McCallum, J. L. McEvoy, T. E. Mirkov, G. Newcombe, J. W. Pscheidt, P. L. Pusey, M. G. Redinbaugh, J. S. Russin, L. W. Timmer, J. Y. Uchida, S. N. Wegulo, F. Workneh, G. Y. Yuen 
Editors of PLANT HEALTH PROGRESS

Editor-in-Chief: T. Murray. Senior Editors: A. M. Alvarez, T. J. Gerik, G. G. Grove, K. Moore, H. V. Morton, S. T. Nameth, M. Putnam, K. K. Rane, K. L. Steffey, Ex officio: A. P. Keinath, R. C. Ploetz, J. B. Ristaino, Staff

\section{Editors of THE PLANT HEALTH INSTRUCTOR}

Editor-in-Chief: G. L. Schumann. M. L. Agnew, C. J. D'Arcy, D. C. Gross, N. Grunwald, M. L. Powelson, D. L. Schadler, D. O. TeBeest, P. Vincelli, Ex officio: D. M. Eastburn, Staff

Phytopathology News Advisory: Chair: S. A. Johnston, J. H. Andrews, A. Grybauskas, D. M. Rizzo, Ex officio: Staff

\section{Society Internal Relations}

Awards and Honors: Chair: L. D. Dunkle. Vice Chair: M. L. Powelson. Immediate Past Chair: G. A. Payne. S. A. Ghabrial, T. R. Gottwald, D. C. Gross, R. C. Rowe

Committee on Committees: Chair: J. H. Andrews, R. R. Belanger, M. A. Ellis

Financial Advisory: Chair: J. L. Sherwood. Treasurer-Elect: E. L. Stromberg, J. H. Andrews, G. C. Bergstrom, J. Fletcher, J. D. MacDonald, M. E. Matheron, R. C. Ploetz

Headquarters Operations: Chair: J. N. BeMiller, J. L. Sherwood, J. Fletcher, G. C. Bergstrom, S. A. Slack, AACC representatives, Ex officio: S. C. Nelson

\section{Society General Policies}

Biotechnology Impact Assessment: Chair: D. C. Sands. Vice Chair: A. O. Charkowski. Immediate Past Chair: R. R. Martin. R. Hull, L. G. Kovacs, V. Kumar, B. Meng, F. W. Nutter, Jr., S. F. Shamoun, P. Warrior, Y. Yang

Collections and Germplasm: Chair: J. P. Wilson. Vice Chair: F. M. Dugan. Immediate Past Chair: M. E. Miller. M. L. Carson, K. M. Crosby, B. J. Goates, E. A. Milus, D. V. Phillips, R. W. Schneider, C. E. Thomas

Cultural Diversity: Chair: D. G. Fernando. Vice Chair: M. Skaria. Immediate Past Chair: R. L. Grayson. T. L. Kirkpatrick

Extension: Chair: W. W. Turacheck. Vice Chair: G. G. Grove. Immediate Past Chair: D. E. Legard. G. S. Bender, R. M. Davis, E. D. De Wolf, A. K. Hagan, G. J. Holmes, T. S. Isakeit, J. M. Leavitt, D. K. Malvick, S. G. P. Nameth, M. A. Newman

Graduate Student: Chair: Z. Kanaan-Atallah. Vice Chair: P. D. Esker. Immediate Past Chair: L. M. Sosnoskie. R. Bulluck, L. N. Caldwell, N. J. Catlin, J. M. Clifford, K. D. Cox, L. E. Davidson, C. L. Lapaire, E. M. Martin, P. R. Northover, M. A. Patil, L. D. Price, J. D. Reddy, S. C. Savchuk, P. Siddavatam, S. Thomas, L. P. Tredway, D. I. Tsitsigiannis

Industry: Chair: C. L. Palmer. Vice Chair: R. Soufi. Immediate Past Chair: K. W. Seebold. D. T. Beadle, C. M. Becker, J. M. Bonman, P. R. Haikal, G. M. Kemmitt, L. B. Laverdiere, M. R. Miles, M. R. Schwarz, Z. Shi, M. G. Tiffany, L. E. Zang

Placement: Chair: A. K. Culbreath. Vice Chair: A. A. Collins. Immediate Past Chair: S. L. Walker. K. W. Campbell, R. Rong, A. K. Wood

Private Practice: Chair: B. Rao. Vice Chair: A. R. Chase. Immediate Past Chair: W. T. Cobb. S. J. Day, J. H. Hart, C. L. Kohls, H. D. Quemada, G. A. Snow, C. S. Thomas

Regulatory Plant Pathology: Chair: K. L. Kosta. Vice Chair: T. N. Boratynski. Immediate Past Chair: S. D. Cohen. A. J. Caesar, T. R. Gottwald, G. Hughes, M. J. Jones, P. I. Lewis, V. S. Malik, C. M. Ocamb, D. C. Opgenorth, G. L. Peterson, E. V. Podleckis, S. A. Rizvi, S. R. Shafer

Teaching: Chair: M. A. Langham. Vice Chair: E. J. Braun. Immediate Past Chair: M. A. Sulzinski. A. B. Baudoin, R. G. Bhat, R. B. Carroll, M. J. Clements, L. Z. De Luna, R. C. Gergerich, K. S. McLean, C. S. Rothrock, R. W. Schneider

Women in Plant Pathology: Chair: P. D. Adams. Vice Chair: TBD. S. M. Bissonnette, S. C. Butterworth, S. Carle, M. K. Hausbeck, L. K. Hawkins, Pl L. Hughes, C. Loschinkohl, M. T. Mmbaga, E. N. Rosskopf, M. Smither-Kopperl

Youth Program: Chair: C. A. Jasalavich. Vice Chair: TBD. Immediate Past Chair: K. Shelton. Y. T. Berthier-Schaad, S. M. Douglas, B. A. Edmunds, W. H. Elmer

\section{Subject Matter}

Bacteriology: Chair: S. M. Brumbley. Vice Chair: TBD. Immediate Past Chair: F. J. Louws. C. T. Bull, W. C. Fuqua, J. S. Hartung, S. Y. He, I.-M. Lee, E. N. Okey, L. S. Pierson, III, B. K. Scholz-Schroeder

Biochemistry, Physiology, and Molecular Biology: Chair: A. F. Bent. Vice Chair: C. B. Lawrence. Immediate Past Chair: T. J. Wolpert. A. M. Fakhoury, T. K. Mitchell, C. M. Okpodu, L. D. Price, R. Rong, L. J. Vaillancourt, Y. Yang

Biological Control: Chair: B. B. McSpadden-Gardener. Vice Chair: E. N. Rosskopf. Immediate Past Chair: W. F. Stoneman. P. D. Adams, S. M. Boyetchko, L. D. Butler, A. J. Caesar, B. K. Duffy, N. I. Kahn, P. D. Kharbanda, B. H. Ownley, C. M. Press, Y. M. Shabana, J. P. Stack, L S. Thomashow, K. R. Vanga, R. Vargas

Chemical Control: Chair: P. M. Brannen. Vice Chair: TBD. Immediate Past Chair: D. B. Langston. R. D. Cartwright, J. P. Damicone, J. E. Fajardo, A. P.
Keinath, R. C. Kemerait, K. W. Seebold, Jr., D. E. Wedge, F. P. Wong

Diagnostics: Chair: L. J. Du Toit. Vice Chair: B. D. Ambruzs. Immediate Past Chair: J. W. Beale. M. Babadoost, E. M. Bentley, J. H. Brock, J. L. Chaky, T. C. Creswell, S. M. Douglas, G. G. Grove, M. Putnam, D. L. Scott

Diseases of Ornamental Plants: Chair: J. W. Buck. Vice Chair: C. Hong. Immediate Past Chair: G. A. Chastagner. A. R. Chase, R. A. Cloyd, M. R. Corral Garcia, J. H. Dobbs, B. A. Edmunds, A. B. Gould, J. L. Hall, M. K. Hausbeck, J. C. Locke, G. W. Moorman, S. G. P. Nameth, A. B. Sinderman, J. Williams-Woodward

Environmental Quality and Plant Health: Chair: S. R. Shafer. Vice Chair: TBD. Immediate Past Chair: C. R. Krause. M. A. Bhatti, J. A. Chavarria, C. L. Lennox, T. S. Schubert, G. S. Smith, S. R. Vann

Epidemiology: Chair: S. J. Pethybridge. Vice Chair: TBD. Immediate Past Chair: K. A. Garrett. E. D. De Wolf, D. G. Fernando, R. J-Garcia, R. Khangura, J. J. Marois, A. L. Mila, F. W. Nutter, P. V. Oudemans, B. M. Wu

Forest Pathology: Chair: G. R. Stanosz. Vice Chair: S. D. Cohen. Immediate Past Chair: L. M. Haugen. P. Bonello, J. N. Bruhn, M. M. Garbelotto, R. S. Hunt, M-S. Kim, N. B. Klopfenstein, G. Laflamme, B. D. Moltzan, S. W. Oak, J. G. O'Brien, C. G. Shaw

Genetics: Chair: S. Kang. Vice Chair: S. Kamoun. Immediate Past Chair: S. E. Gold. R. G. Bhat, R. L. Bowden, X. Chen, G. W. Douhan, M. Garcia-Pedrajas, A. E. Glenn, Y. Jin, J. S. Lehman, F. N. Martin, G.-L. Wang

Host Resistance: Chair: J. A. Kolmer. Vice Chair: M. R. Miles. Immediate Past Chair: A. E. Dorrance. M. J. Clements, C. S. Kousik, F. N. Lee, H. K. Ngugi, J. L. Norelli, B. A. Perez, M. G. Redinbaugh, R. W. Schneider, C. E. Thomas, C. M. Vera Cruz, X. Zheng

Integrated Pest Management: Chair: L. Fought. Vice Chair: D. S. Egel. Immediate Past Chair: S. A. Alexander. W. M. Brown, Jr., A. K. Culbreath, K. L. Everts, S. A. Heuchelin, C. L. Kohls, K. L. Pernezny, J. L. Riggs, P. D. Roberts, H. Scherm, S. F. Shamoun, E. J. Sikora

Mycology: Chair: W. Chen. Vice Chair: S. B. Goodwin. Immediate Past Chair: C. M. Stiles. D. M. Beyer, R. Blanco Pietro, M. L. Boyd, D. B. Chalkey, J. C. Correll, S. Demir, D. M. Eastburn, D. A. Glawe, J. L. Kerrigan, C. L. Lapaire, M. E. Palm, L. Wasilwa

Mycotoxicology: Chair: A. E. Desjardins. Vice Chair: G. Kuldau. Immediate Past Chair: G. P. Munkvold. R. L. Brown, R. Dill-Macky, G. A. Gilbert, G. N. Odvody, D. G. Panaccione, R. H. Proctor, A. Schaafsma, D. A. Schisler, D. I. Tsitsigiannis, G. L. Windham

Nematology: Chair: S. Chen. Vice Chair: TBD. Immediate Past Chair: W. T. Crow. R. F. Davis, T. L. Kirkpatrick, S. A. Lewis, A. E. MacGuidwin, J. P. McMahon, G. L. Tylka

Pathogen Resistance: Chair: H. L. Ypema. Vice Chair: K. L. Stevenson Immediate Past Chair: T. B. Brenneman. T. Chand-Goyal, A. M. Diner, M. T. McGrath, S. M. Schneider, W. F. Wilcox, J. A. Yocum

Phyllosphere Microbiology: Chair: C. C. Mundt. Vice Chair: W. F. Mahafee. Immediate Past Chair: S. Lindow. O. Carisse, V. J. Elliott, B. J. Jacobsen, P. L. Pusey, B. D. Shaw, G. Y. Yuen

Plant Disease Losses: Chair: S. E. Penix Fitzsimmons. Vice Chair: D. A. Shah. Immediate Past Chair: W. W. Turechek. J. Guan, G. Hughes, J. E. Lundquist, L. V. Madden, M. H. Royer

Plant Pathogen and Disease Detection: Chair: N. L. Robertson. Vice Chair: P. Ellis. Immediate Past Chair: C. A. Levesque. J. J. Beck, R. M. DeVriesPaterson, R. Frederick, N. Glynn, D. G. Luster, A. Rowhani, M. A. Sulzinski, I. E. Tzanetakis, E. Vivoda, J. Q. Xia

Postharvest Pathology: Chair: W. J. Janisiewicz. Vice Chair: J. L. McEvoy. Immediate Past Chair: W. S. Conway. J. E. Adaskeveg, J. A. Bartz, B. D. Bruton, G. J. Holmes, E. M. Imai, T. J. Michailides, D. Prusky, P. G. Sanderson, P. L. Sholberg, J. L. Smilanick, D. Sugar, D. C. Thompson, C. Xiao, J. Zhang

Seed Pathology: Chair: L. M. Shepherd. Vice Chair: B. L. Randall-Schadel. Immediate Past Chair: C. J. Kurowski. S. Autrey, C. C. Block, P. Fenn, K. D. Gwinn, P. T. Himmel, S. M. Koehler, T. A. Kucharek, M. E. Meadows

Soil Microbiology and Root Diseases: Chair: J. H. Bowers. Vice Chair: J. L. Parke. Immediate Past Chair: M. L. Smither-Kopperl. J.C. Bienapfl, R. Bulluck, C. H. Canady, C. G. Eayre, W. H. Elmer, L. E. Hanson, M. S. Krause, J. E. Kurle, P. Lemanceau, R. J. McGovern, M. Raaijmakers, M. S. Reddy, B. Spakes Richter

Tropical Plant Pathology: Chair: S. S. Savary. Vice Chair: TBD. Immediate Past Chair: J. V. Da Graca. L. A. Calvert, M. P. Grisham, N. A. Harrison, S. Kelemu, D. N. Kuhn, J. O. Kuti, R. G. McGuire, F. M. Ochoa, J. J. Ooka, R. P. Rodriguez, R. A. Valverde, A. Westphal, J. P. Wilson

Turfgrass Pathology: Chair: S. B. Martin. Vice Chair: L. P. Tredway. Immediate Past Chair: L. J. Stowell. M. L. Agnew, L. E. Datnoff, S. P. Falk, K. J. Gard, D. Y. Han, G. Jung, B. Rao, M. R. Schwarz, W. Uddin, N. R. Walker

Virology: Chair: J. E. Schoelz. Vice Chair: J. K. Brown. Immediate Past Chair: J. F. Murphy. S. T. Adkins, J. M. Anderson, R. H. Brlansky, M. E. Hilf, A. V. Karasev, R. C. Larsen, L. Lee, H. Liu, G. Loebenstein, C. J. M. Maroon, B. Meng, J. E. Polston, W. Qiu, B. R. Reddick, D. C. Stenger, J. Verchot Lubicz 